\title{
GANANCIA INICIAL DE PESO DEL RECIÉN NACIDO DE TÉRMINO HIJO DE MADRE ADOLESCENTE
}

\author{
EARLY WEIGHT GAIN OF INFANTS BORN \\ TO ADOLESCENT MOTHERS
}

\author{
Carla Zapata G., Carlos Castillo D. \\ Departamento de Pediatría, Facultad de Medicina, Campus Centro, \\ Universidad de Chile. Santiago, Chile.
}

\begin{abstract}
Background: Adolescent pregnancy is frequent in Chile, and their infants could present an increased risk of inadequate breast feeding and weight gain. Objectives: To assess the early weight gain in infants born to Chilean adolescent mothers and their association with breast feeding, risk of jaundice and hospitalization. Subjects and methods: Forty full-term and adequate for gestational age newborns (SG; M/F : 27/13), born to healthy adolescent mothers (less than $<19$ y at delivery) (SG) from low socioeconomic groups, were recruited in a public hospital in Santiago, Chile. They were paired with 36 born to healthy primiparous adult mothers (control group CG; M/F :19/17); those born with low birth weight or congenital malformations were excluded. They were assessed at well baby clinics in primary care centers for weight, length, (median age: 8 and 30 d.; breast feeding, jaundice and hospitalization were registered). Results: No significant differences were found in weight gain at 8th day of age (SG: $-1,5 \mathrm{~g} / \mathrm{d}(-107$ to +36$) ; C G-5,7 \mathrm{~g} / \mathrm{d}(-59$ to +39$)(\mathrm{NS})$, both lower than the weight increments of WHO standards (14-21 g/d); birth weight recovery was found in SG: $52 \% \%$ and CG: $44 \%$ (NS). Neither a difference was found at 30 days (SG: 31g/d (-1.7 to +79), CG: 28 g/d (-3 to63); (NS), comparable to WHO standards (males: $34 \mathrm{~g} / \mathrm{d}$; females: $29 \mathrm{~g} / \mathrm{d}$ ). However, the sub-group of infants born to adolescent mothers 15-16 y. presented a greater weight gain than those to adolescents mothers 16-17 y. $(38 \mathrm{~g} / \mathrm{d}(9$ to $79 \mathrm{vs} 25 \mathrm{~g} / \mathrm{d},-1,7$ to +57$)$ than the CG $(p<0.02)$. Formula breast feeding was provided to $8 / 40$ in $S G$ and 10/36 in CG (NS). No differences in jaundice was found at the 3rd day, but an increased rate was observed at the 8th day (SG 13/40 vs CG2/36) (OR 8.2; CI: 1.7-39; $p=0.003)$, without an increased risk of hospitalization. Conclusions: Infants born to Chilean adolescent mothers have a comparable weight gain to infants born to adult mothers, with a comparable time of birth weight recovery. Those born to mothers 15-16 years of age had a greater weight gain than those born to mothers 17-18 years or adult mothers. Jaundice to the $8^{\circ}$ day was greater in the $S G$ with no increased rate of hospitalization.
\end{abstract} They present a good rate of breast feeding.

Key words: adolescent mothers, infants, early weight gain, birth weight recovery.

Este trabajo fue recibido el 25 de Julio de 2011 y aceptado para ser publicado el 25 de Marzo de 2012.

\section{INTRODUCCIÓN}

Ante las demostradas ventajas de la lactancia materna, la OMS recomienda como alimentación óptima una lactancia materna exclusiva durante los primeros 6 meses de vida (1-2). Una parte importante del éxito en la lactancia materna se debe a factores presentes los primeros días de vida, asociado a buenas prácticas de apego (3-5).

El recién nacido de término normalmente pierde hasta un 7-10\% del peso de nacimiento en los primeros días, a expensas del volumen extracelular, debiendo recuperar su peso de nacimiento antes de los 10 días (6-8). Según las nuevas referencias de OMS se espera un incremento promedio en torno a $40 \mathrm{~g} /$ día, desde la recuperación del peso de nacimiento, durante el primer mes en recién nacidos alimentados al pecho en forma exclusiva. Si la subdividimos por sexo el incremento desde 0-30 días es $29 \mathrm{~g} / \mathrm{d}$ en la mujer y $34 \mathrm{~g} / \mathrm{d}$ en el 
hombre (p50) según las referencias OMS (9). La pérdida de peso mayor del $10 \%$ o la lenta recuperación del peso de nacimiento puede deberse a hipoalimentación por problemas de técnica de lactancia o una manifestación de enfermedades asociadas (8). Una consecuencia de esto es la hiperbilirrubinemia, cuyo mecanismo probable es el aumento de la circulación enterohepática por disminución de la motilidad intestinal, a lo que se agrega algún grado de deshidratación (10-11).

El embarazo en la adolescencia es un problema de salud pública importante, observándose con mayor frecuencia en los sectores socioeconómicos más pobres. Se estima que en los países en desarrollo el 20-60\% de estos embarazos son no deseados, afectando el nivel de escolaridad de las adolescentes, tienen mayor dificultad para conseguir trabajo, abandono por su pareja, todo lo cual acentúa el riesgo de pobreza (12).

La prevalencia de embarazo adolescente varía en diferentes países, se estima en $12-13 \%$ en países desarrollados como EEUU y $14-20 \%$ en países menos desarrollados como Chile (13). En comunidades más pobres de Santiago puede llegar hasta un 15\% del total de los embarazos. En el Servicio de Salud Metropolitano Central es de 10,4\% según cifras del MINSAL 2004.

El embarazo en la adolescencia se ha asociado a mayores complicaciones durante su curso y en el parto, con un mayor riesgo de parto prematuro y bajo peso de nacimiento (14); lo que podría ser explicado por la competencia de nutrientes que se produce entre la madre adolescente en crecimiento y el feto en formación (15).

Nuestra hipótesis es que los recién nacidos hijos de madre adolescente tienen un riesgo aumentado de una menor velocidad de recuperación del peso de nacimiento. El objetivo de este estudio fue determinar el tiempo de recuperación del peso de nacimiento en recién nacidos de madres adolescentes chilenas, estudiar la ganancia de peso durante el primer mes y analizar la asociación entre ganancia de peso con abandono de lactancia materna, riesgo de ictericia y de hospitalización.

\section{SUJETOS Y MÉTODOS Sujetos}

El grupo de estudio estuvo constituido por RN de término nacidos en la maternidad del Hospital San Borja Arriarán, correspondiente al Servicio de Salud Metropolitano Central hijos de primíparas adolescentes ( $\leq 19$ años al parto), sin malformaciones congénitas o enfermedades evidentes. El grupo control lo constituyeron $\mathrm{RN}$ de primíparas $\geq 19$ años con características comparables de control: escolaridad materna (años de estudio), estrato socioeconómico (quintil de ingreso), tipo de parto y en sus hijos: el peso y talla de nacimiento y la edad gestacional.

Los criterios de exclusión fueron: ser recién nacidos de pretérmino, asfixia perinatal, incompatibilidad de grupo, malformaciones mayores, hipotiroidismo u otras alteraciones metabólicas; madres con diabetes gestacional $\mathrm{u}$ otra enfermedad metabólica.

Tamaño muestral: No conocemos de trabajos actuales acerca de la diferencia de recuperación de peso de nacimiento de los $\mathrm{RN}$ de madres adolescentes en relación a madres primíparas no adolescentes. Para evaluar las diferencias en los incrementos de peso del primer mes nos hemos basado en los incrementos mensuales de los estándares OMS 2006 que son en promedio 40 g/día en recién nacidos alimentados al pecho; hemos asumido de esos datos una desviación estándar de 7 g/día y en base a estudios anteriores basados en lactancia artificial preferente (7). Estimando una diferencia en el incremento de peso diario de $0,5 \mathrm{DE}$ entre ambos grupos $(3,5 \mathrm{~g})$, con un error alfa de 0,05 y un poder de $80 \%$ se estimó un tamaño muestral de 34 sujetos para cada grupo.

En un estudio de caso control anidado en una cohorte, se reclutaron 76 madres y sus hijos del puerperio del Hospital San Borja Arriarán desde agosto 2007 a agosto 2009. La selección de controles se realizó de entre aquellos que nacieron el mismo día y comparables al caso.

Previo consentimiento escrito e informado a la madre, se recolectaron los datos mediante visita a puerperio hasta el momento de alta y con contacto telefónico posterior, donde se consultó por el peso y la talla registrado en el carnet de control sano de su consultorio de atención primaria, enfermedades, coloración de la piel, hospitalizaciones y tipo de alimentación, registrándose los datos en una ficha clínica estructurada.

El análisis estadístico incluyó: promedios, medianas, prueba t-student, ANOVA, Kruskal-Wallis para ver significación estadística entre las velocidades de aumento de peso de ambos grupos; chi-cuadrado para frecuencia de ictericia al $3^{\circ}$ y $8^{\circ}$ día, razones de probabilidad (OR) para riesgo de incorporar fórmula artificial y riesgo de ictericia. Análisis de regresión múltiple para evaluar el efecto de variables de control: como el tipo de parto, y la educación materna.

\section{RESULTADOS}

Se reclutaron 76 madres con sus recién nacidos, 40 $\mathrm{RN}$ de madres adolescentes primíparas < 19 años (GE; H/M: 27/13) y $36 \mathrm{RN}$ de madres adultas primíparas (GC; H/M:19/17). Ambos grupos fueron comparables en nivel socioeconómico, tipo de parto, edad gestacional, peso de nacimiento (tabla 1). No fueron comparables en edad y años de estudio que era lo esperable, por tratarse de adolescentes que aún no han completado sus estudios y 
muchas no continuaran estudiando.

En la evaluación de peso post- alta, se observó una dispersión en los días del control con promedio de $9 \pm$ 3 días en el grupo control y $8 \pm 4$ días en el grupo de madres adolescentes. Al $8^{\circ}$ día se observó una ganancia de peso de GE -1,5 g/día (-107 a +36 g/día); varones: $-2,7$ g/día (-107,5 a 36 g/día) y mujeres 1 g/día (-50 a 33 g/día) (NS); GC -5,7 g/día (-59 a +39 g/día): varones: -6 g/día (-50 a 26,4 g/día) y mujeres $-5,45 \mathrm{~g} /$ día (-59 a $39 \mathrm{~g} /$ día), diferencia no significativa ni tampoco con el grupo GE, ambos grupos presentaban un ritmo bajo lo esperado por OMS (14-21 g/día); una alta proporción no habían recuperado peso de nacimiento al $8^{\circ}$ día: GE: 48\%, GC: 56 (NS). Al evaluar el incremento de peso al

\begin{tabular}{|c|c|c|c|}
\hline \multicolumn{4}{|c|}{$\begin{array}{l}\text { TABLA 1 } \\
\text { Características de las madres adolescentes y adultas }\end{array}$} \\
\hline & $\begin{array}{c}\text { Madres adolescentes } \\
\text { (40) }\end{array}$ & $\begin{array}{c}\text { Madres no adolescentes } \\
\text { (36) }\end{array}$ & $\begin{array}{l}\text { Significación } \\
\text { estadística }\end{array}$ \\
\hline Edad $(\mathrm{x} \pm \mathrm{DE})$ & $16 \pm 1$ & $24 \pm 4$ & \\
\hline Educación (años) & $10 \pm 1,6$ & $12,2 \pm 1,6$ & \\
\hline Ingreso per capita (frec. Quintiles $1,2,3$ ) & $40 / 40$ & $27 / 29$ & NS \\
\hline Tipo de parto $(\mathrm{V} / \mathrm{C})^{*}$ & $32 / 8$ & $25 / 11$ & NS \\
\hline
\end{tabular}

\section{TABLA 2}

Características de los recién nacidos

\begin{tabular}{|c|c|c|c|}
\hline & $\begin{array}{l}\text { Hijos de madres adolescentes } \\
\qquad(n=40)\end{array}$ & $\begin{array}{l}\text { Hijos de madres adultas } \\
\qquad(\mathrm{n}=36)\end{array}$ & \\
\hline Peso de nac. (g) & $3337+/-422$ & $3453+/-318$ & NS \\
\hline Talla de nac. $(\mathrm{cm})$ & $50+/-1,7$ & $50+/-2,4$ & NS \\
\hline Edad gestac. $(\mathrm{sem})^{*}$ & $39(38-40)$ & $39(37-41)$ & NS \\
\hline Peso egreso hosp.(g) & $3174+/-386$ & $3241+/-311$ & NS \\
\hline Peso $8^{\circ}$ día (g) & $3380+/-446$ & $3439+/-385$ & NS \\
\hline$\Delta$ Peso $0-8$ días $(\mathrm{g} / \mathrm{d})^{*}$ & $-1,5(-107 \mathrm{a}+36)$ & $-5,7(-59$ a 39$)$ & NS \\
\hline Varones (g/d) & $-2,7(-107$ a 36$)$ & $-6,2(-50$ a 26,4$)$ & NS \\
\hline Mujeres (g/d) & $1(-50$ a 33$)$ & $-5,4(-59$ a 39$)$ & NS \\
\hline Peso 30 días (g) & $4383+/-751$ & $4418+/-703$ & NS \\
\hline$\Delta$ Peso $0-30$ días $(\mathrm{g} / \mathrm{d})^{*}$ & $31(-1,7 \mathrm{a}+79)$ & $28(-3$ a 63$)$ & NS \\
\hline$\Delta$ Peso varones $(\mathrm{g} / \mathrm{d})$ & 31 (0 a 79) & $29,4(-3,3$ a 63$)$ & NS \\
\hline$\Delta$ Peso mujeres $(\mathrm{g} / \mathrm{d})$ & $30(-1,7$ a 57$)$ & $26(4,3$ a 50$)$ & NS \\
\hline Día control mes & $35+/-9$ & $34+/-8$ & NS \\
\hline Día control $8^{a}$ & $8,5 \pm 4$ & $9 \pm 2,6$ & NS \\
\hline Ictericia 3er día & $12 / 40$ & $7 / 36$ & NS \\
\hline Ictericia $8^{\circ}$ día & $13 / 40$ & $2 / 36$ & OR: 8,2 p 0,003 \\
\hline Lactancia artificial & $8 / 40$ & $10 / 36$ & NS \\
\hline
\end{tabular}


mes de vida no se encontró diferencias significativas entre los grupos ( GE 31g/día; -1,7 a 79, GC 28 g/día; -3 a $63 \mathrm{~g} /$ día NS), sin diferencia entre sexos (GE hombres 31g/día (0 a 79g/día) y mujeres $30 \mathrm{~g} /$ día (-1,7 a 57 g/ día) GC; hombres 29,4g/día (-3 a 63 g/día) y mujeres $26 \mathrm{~g} /$ día (4 a 50g/día), con tendencia a un mayor incremento diario en el grupo de madres adolescentes ( NS) (tabla 2). Al subdividir el grupo de madres adolescentes en tramos de edad de 15-16 años (n:20) y 17-18 años (n:20), el incremento promedio diario en el primer mes de vida fue significativamente mayor en el subgrupo de 15-16 años que en el sub-grupo de 17-18 años, 38g/día (9 a 79 g/día) en el grupo de 15-16 años y 25 g/día (-1,7 a $57 \mathrm{~g} /$ día $)(\mathrm{p}<0,02)$. También fue significativamente mayor al compararlos con GC $(\mathrm{p}<0,02)$ (tabla 3$)$. No hubo diferencias significativas al comparar estos 2 subgrupos en el incremento diario hasta el $8^{\circ}$ día $(p=0,6)$.

En relación al riesgo de hospitalización, no se registraron hospitalizaciones en el grupo de madres adolescentes y se registró en 2 de 29 en el grupo de madres no adolescentes (NS). Ambas hospitalizaciones fueron por ictericia, en una de los cuales además se pesquisó una cardiopatía congénita simple.

No hubo diferencias en el riesgo de presentar ictericia entre los grupos al $3^{\circ}$ día de vida: 12/40 recién nacidos GE y 7/36 recién nacidos GC (NS). Se observó un mayor riesgo de presentar ictericia al $8^{\circ}$ día en los hijos de madres adolescentes, que en los hijos de madres adultas (13/40 vs 2/36, OR: 8,2 IC: 1,7-39; p 0,003). La presencia de ictericia se asoció a mal incremento del peso con retraso en la recuperación de peso de nacimiento al control del $8^{\circ}$ día con recuperación del incremento a los 30 días en 5/13 de recién nacidos en GE y 1/2 recién nacidos del GC. Sólo un recién nacido del grupo de madres adolescentes presenta incompatibilidad de grupo clásico, sin necesidad de hospitalización por su ictericia.

No hubo diferencias en la incorporación de lactancia artificial antes del mes de vida: 8/40 hijos de madres adolescentes vs 10/36 hijos de madres adultas (OR: 0,65; IC $0,22-1,88, \mathrm{NS}$ )

\section{DISCUSIÓN}

A diferencia de lo planteado en nuestra hipótesis no encontramos diferencias estadísticas en la recuperación del peso de nacimiento e incremento de peso a los 30 días en los recién nacidos hijo de madres adolescentes en comparación con recién nacidos de madres primíparas no adolescentes, lo que podría ser esperable en el grupo de 17 a 18 años que ya han completado su desarrollo físico y sexual, pero llama la atención en el grupo de 15 a 16 años en que se podría esperar que aún no hayan completado su desarrollo potencial y pudiese haber una competencia por los nutrientes como lo planteado por Scholl y col (15). Además, se encontró que el incremento diario al mes de vida en el subgrupo de madres adolescentes de 15 a 16 años, era $>1 \mathrm{DE}$ según las últimas tablas de OMS (: hombre 34 g/día: mujer 29 g/día), en contraste con los $25 \mathrm{~g} /$ día del sub-grupo de 17 a 18 años (9) . No está claro el motivo de esta diferencia; pensamos que pudiera estar participando una mayor frecuencia de exposición a drogas, mayor deserción escolar y mayor vulnerabilidad social en el grupo de madres de 17 a 18 años, como ya está planteado por Padilla (12) y Donoso (21) en su revisión, donde considera como factor importante, el bajo nivel socioeconómico y la edad en relación a mortalidad neonatal y postnatal.

De las adolescentes evaluadas 11 se reconocían conviviendo al momento del parto de las cuales 6 correspondían al grupo de 15 a 16 años (55\%) y 5 del grupo 17 a 18 años (45\%), de las adolescentes que convivían 6/11 (55\%), tenían un incremento de peso al mes >1DS de las tablas de OMS. Al considerar que sus condiciones sociales son comparables, podríamos decir que los factores biológicos dados por la edad no influirían en

\section{TABLA 3}

Pesos e incrementos de peso de hijos de madres adolescentes por grupos etarios vs madres adultas

\begin{tabular}{|lcccc|} 
& \multicolumn{2}{c}{ Madres adolescentes } & Madres adultas & p \\
& $\mathbf{1 4 - 1 6}$ años & $\mathbf{1 7 - 1 8}$ años & & \\
\hline Peso 8 día (g) & $3444 \pm 489$ & $3322 \pm 407$ & $3439 \pm 385$ & $\mathrm{NS}$ \\
Incremento 0-8 d (g/d) & $0,77(-107$ a 36) & $-3,6(-50$ a 33) & $-5,7(-59$ a 39) & NS \\
Peso 30 días (g) & $4593 \pm 795$ & $4175 \pm 651$ & $4418 \pm 703$ & NS \\
Incremento 0-30 d (g/d) & $38(9$ a 79) & $25(-1,7$ a 57) & $28(-3$ a 63) & $\mathrm{P}=0,02$ \\
\hline * test ANOVA Kruskal-Wallis & & & \\
\hline
\end{tabular}


el periodo post natal produciéndose una adaptación fisiológica de la adolescente a esta situación, quizás una sobre-adaptación si lo comparamos con las adolescentes mayores (17 a 18 años) y las adultas.

Se han realizado estudios comparativos en adolescentes embarazadas, puérperas y adultas, con marcadores del metabolismo óseo, relación calcio -fósforo y densitometría ósea, llegándose a la conclusión que se producen algunos cambios funcionales de adaptación mas marcados en adolescentes que favorecen el ahorro de calcio durante el embarazo (19-20).

Otro factor a considerar que pudiese sesgar la evaluación es el hecho de desconocer el estado nutricional de las madres tanto adolescentes como del grupo control previo al embarazo, buscando signos de malnutrición previa. Existen reportes en la literatura en relación al riesgo nutricional en madres adolescentes tanto de malnutrición por déficit o exceso dependiendo del nivel socioeconómico y del nivel de desarrollo del país, pero no existen publicaciones relacionadas con déficit específicos en el lactante (16-17).

En relación al riesgo de complicaciones y hospitalización, no se encontró mayor riesgo en el GE, no concordante con publicaciones de estudios retrospectivos donde se ha encontrado aumento del riesgo de mortalidad post natal en hijos de madres adolescentes con grupos comparables en NSE, edad gestacional, incremento de peso durante el embarazo, raza, nivel educacional sin poder explicar con estos factores tal diferencia (18-21).

Respecto al objetivo de evaluar el riesgo de ictericia, destaca el mayor riesgo de ictericia al $8^{\circ}$ día en el grupo de madres adolescentes, pero sin riesgo de hospitalización, asociándose la presencia de ictericia a un menor incremento de peso principalmente al control del $8^{\circ}$ día con recuperación del incremento al control del mes, sin diferir en lo descrito en la literatura donde no se considera al recién nacido de madre adolescente con mayor riesgo de hiperbilirrubinemia severa, si no que se asocia a parto prematuro y bajo peso de nacimiento principalmente, que en este caso se excluyeron de la muestra.

Destaca una buena adherencia de las madres de ambos grupos a la lactancia materna predominante durante el primer mes de vida, lo que puede estar relacionado al hecho que en general el tiempo de recuperación del peso de nacimiento fue adecuado en ambos grupos, con buen incremento al primer mes, sin embargo es una conclusión apresurada en el seguimiento de un mes.

Si bien el embarazo adolescente se relaciona a mayor riesgo de complicaciones durante el embarazo como parto prematuro, RCIU y PEG, no fue posible demostrar riesgo post-natal de déficit nutricional durante el primer mes de vida, hiperbilirrubinemia severa, hospitalizacio-

\section{FIGURA 1}

Aumento de peso de los lactantes promedio 0-30 días (g/día), según subgrupos de madres adolescentes (15 a 16 años y 17 a 18 años) y madres adultas.

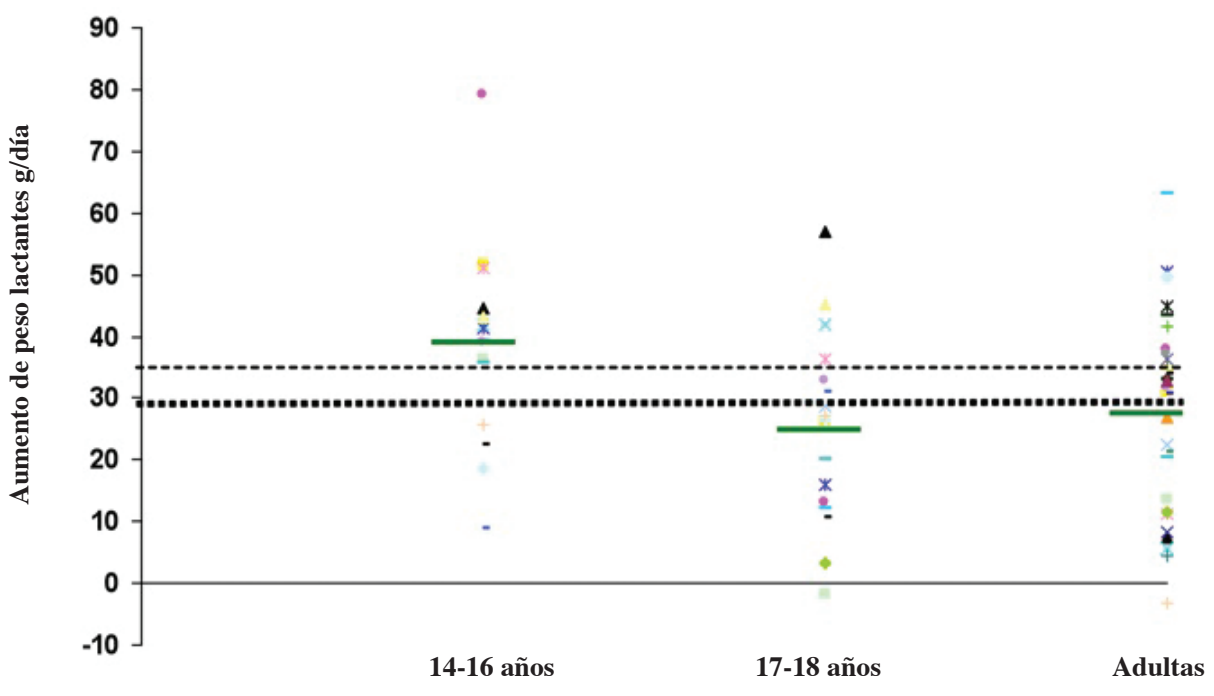

EDAD DE LAS MADRES 
nes o mala adherencia a lactancia materna. Sin embargo la deserción escolar temprana (solo el 50\% se reconocía estudiante al momento del parto), con la consiguiente baja escolaridad son factores de importancia en perpetuar el círculo de pobreza, que a la larga aumentaran el riesgo de estos niños de problemas de mal nutrición entre otros.

Se debe considerar además que el seguimiento fue sólo un mes por lo que estos mismos parámetros evaluados podrían cambiar en un seguimiento a 6 meses o 1 año. Dada la asociación entre ganancia precoz aumentada de peso en lactantes y riesgo de obesidad en edades posteriores (22), dentro de la variabilidad observada en las ganancias de peso debiera seguirse con atención a aquellos lactantes que presentan una ganancia acelerada y precoz de peso.

En resumen, los hijos de madres adolescentes chilenas controlados en el sistema público de salud presentan un incremento de peso semejante durante el primer mes de vida al de hijos de madres primíparas adultas; el subgrupo de hijos de madres adolescentes con edades entre 15 y 16 años presentan un incremento de peso mayor al de hijos de madres de 17 a 18 años y al de madres adultas. Una alta proporción de niños no han recuperado el peso de nacimiento al $8^{\circ}$ día de vida.

\section{RESUMEN}

Introducción: el embarazo de la adolescente es frecuente en Chile; sus hijos pueden tener mayor riesgo de lactancia materna insuficiente de cuidados inadecuados y mal incremento ponderal. Objetivo: Estudiar la ganancia de peso durante el 1er mes de vida de recién nacidos (RN) hijos de madres adolescentes y su asociación con riesgo de ictericia, hospitalización y abandono de lactancia materna. Sujetos y métodos: Se estudiaron 40 RN de término, hijos de madres primíparas adolescentes $\leq$ 19 años, de estratos socioeconómicos bajos, adecuados para la edad gestacional (AEG), sin patología aguda o malformaciones, (GE; H/M: 27/ 13) y se compararon con $36 \mathrm{RN}$ de madres adultas primíparas (GC; H/M: 19/17), de un hospital público de Santiago. Fueron controlados en atención primaria (peso, talla) con mediana a los 8 y 30 días en ambos grupos, se consultó por ictericia, lactancia materna y hospitalización. Resultados: Al $8^{\circ}$ día la ganancia de peso fue: GE -1,5 g/día (-107 vs $+36)$ vs GC $-5,7 \mathrm{~g} / \mathrm{d}(-59 \mathrm{a}+39)(\mathrm{NS}),<\mathrm{al}$ incremento esperado por OMS (14-21g/d), habían recuperado peso de nacimiento $52 \%$ de GE y $44 \%$ GC (NS). Al mes no hubo diferencias en el incremento diario (GE 31; -1,7 a 79 g/d, GC 28; -3 a 63 g/d NS), semejantes a estándares OMS (x: $34 \mathrm{~g} /$ día en hombres y $29 \mathrm{~g} /$ día en mujeres). Al subdividir GE en grupos de madres: de 15 a 16 y de 17 a 18 años, se observó un mayor incremento diario el 1er mes en el grupo de 15 a 16 años: $38 \mathrm{~g} / \mathrm{d}(9-79)$ vs 17 a 18 años: $25 \mathrm{~g} / \mathrm{d}(-1,7 \mathrm{a} 57)(\mathrm{p}<0,02)$ y con el GC $(\mathrm{p}<0,02)$. No hubo diferencias en el riesgo de ictericia al $3^{\circ}$ día: GE:12/40, GC: 7/36 (NS), pero hubo diferencia al $8^{\circ}$ día, GE: 13/40, GC 2/36 (OR: 8,2 IC:1,7-39; p=0,003), sin mayor riesgo de hospitalización. Ambos grupos recibieron lactancia materna, con fórmula artificial en GE: 8/40, GC: 10/36 (NS). Conclusiones: Los hijos de madre adolescentes no presentan mayor riesgo de retraso en la recuperación de $\mathrm{PN}$ comparados con hijos de madres primíparas adultas, el sub-grupo de hijos de adolescentes de 15 a 16 años tuvieron un mayor incremento de peso que el de 17 a 18 años y el GC. Si bien el riesgo de ictericia al $8^{\circ}$ día es mayor en el GE, este no constituyó un factor de hospitalización. Presentan buena adherencia a lactancia materna.

Palabras clave: madres adolescentes; hijos; ganancia precoz de peso; recuperación peso nacimiento.

Dirigir la correspondencia a:

Dra. Carla Zapata G.

Departamento de Pediatría

Facultad de Medicina Campus Centro

Universidad de Chile

Santa Rosa 1234

Santiago, Chile

Teléfono: 5748806

E-mail: carlazapatag@yahoo.com

\section{BIBLIOGRAFÍA}

1. WHO. Community-based strategies for breastfeeding promotion and support in developing countries, Geneva, 2003

2. Chandran L, Gelfer P. Breastfeeding: The essential principles Pediatr Rev 2006. 11· 409-17.

3. Mikiel-Kostyra K, Mazur J, Bołtruszko I. Effect of early skin-to-skin contact after delivery on duration of hreastfeeding. a prospective cohort study. Acta Paediatr 2002. 91.1288-9

4. Vaidya K, Sharma A, Dhungel S. Effect of early mother-baby close contact over the duration of exclusive breastfeeding. Nepal Med Coll J 2005; 7:138-40.

5. Yoshitada Y, Itsuro Y. Breast-feeding frequency during the first $24 \mathrm{hr}$ after birth in fullterm neonates. Pediatrics 1990; 86:171-5.

6. Macdonald P D, Ross S R M, Grant L; Young D. Neonatal weight loss in breast and formula fed infants. Arch Dis Child Fetal Neonat Ed. 2003; 88: 472-6.

7. Guo SM, Roche AF, Fomon SJ, Nelson SE, Chumlea WC, Rogers RR, et al. Reference data on gains in 
weight and length during the first two years of life. J Pediatr 1991; 119:355 - 62.

8. Covas M., Alda E., Ventura S. Variación del peso durante el primer de vida en recién nacidos de término sanos con lactancia materna exclusiva. Arch Argent Pediatr 2006; 104:399-405.

9. World Health Organization, Department of Nutrition for Health and Development .WHO growth standards, 2006.

10. Maisels MJ, Gifford K. Breast feeding, weight loss and jaundice. J Pediatr 1983; 102:117-8.

11. Michael L. Moritz, Mioara D. Manole, Debra L. Bogen and J. Carlos Ayus. Breastfeeding-associated hypernatremia: are we missing the diagnosis?. Pediatrics 2005; 116:e343-e 7.

12. Padilla de Gil: Aspectos médicos y sociales de la maternidad en la adolescencia. Rev Sogia 2000; 7: $16-25$.

13. Servicio Nacional del Menor (SERNAM). Anuario de Bioestadísticas 2004.

14. Jolly MC, Segire N, Harris J, Robinson S, Regan L: Obstetric risks of pregnancy in women less than 18 years old. Obstet Gynecol 2000; 96: 962-6.

15. Scholl TO, Hediger ML, Ances IG. Maternal growth during pregnancy and decreased infant birth weight Am J Clin Nutr 1990; 51:790-3.
16. A Roberto Frisancho, $\mathrm{PhD}$, Jorge Matos, $\mathrm{MD}$, and Pam Flegel, BA. Maternal nutritional status and adolescent pregnancy outcome. Am J Clin Nutr 1983; 38:739- 46.

17. Moran VH. A systematic review of dietary assessments of pregnant adolescents in industrialized countries. Br J Nutr 2007; 97:411-25.

18. Chen X, Wen SW, Fleming N, Yang Q, Walker M. Increased risks of neonatal and postneonatal mortality associated with teenage pregnancy had different explanations. J Clin Epidemiol 2008; 61: 688-94.

19. BezerraF, Laboissierère F, King J, Donangelo C. Pregnancy and lactation affect markers of calcium and bone metabolism differently in adolescent and adult women with low calcium intake. J Nutr 2002; 132: 2183-7.

20. Chantry C, Auinger P, Byrd R. Lactation among adolescent mother and subsequent bone mineral density.

21. Donoso Siña E, Becker Valdivieso J, Villarroel de Pino L. Birth rates and reproductive risk in adolescents in Chile, 1990-1999. Rev Panam Salud Publ 2003; 14:3-8.

22. Wijlaars LP, Johnson L, van Jaarsveld CH, Wardle J. Socioeconomic status and weight gain in early infancy. Int J Obes (Lond) 2011; 35: 963-70. 